\title{
Effects of vascular endothelial growth factor and insulin growth factor-1 on proliferation, migration, osteogenesis and vascularization of human carious dental pulp stem cells
}

\author{
WANYU LU, WENAN XU, JIANJIA LI, YAN CHEN, YUHUA PAN and BULING WU
}

Department of Stomatology, Nanfang Hospital, Southern Medical University, Guangzhou, Guangdong 510515, P.R. China

Received March 1, 2019; Accepted July 15, 2019

DOI: $10.3892 / \mathrm{mmr} .2019 .10606$

\begin{abstract}
The present study aimed to investigate the effects of vascular endothelial growth factor (VEGF) and insulin-like growth factor-1 (IGF-1) on the proliferation, migration and differentiation of human carious dental pulp stem cells (hCDPSCs), and to elucidate the underlying mechanism(s). Cell counting kit- 8 assay was used to detect the effect of different concentrations of IGF-1 and VEGF on the proliferation of hCDPSCs. Transwell assay was used to detect the migratory ability of the hCDPSCs. Alizarin red and alkaline phosphatase (ALP) staining were used to detect the osteogenic ability of hCDPSCs, whereas the angiogenic ability of the hCDPSCs was tested by tube formation assay. Reverse transcription-quantitative polymerase chain reaction (RT-qPCR) and western blotting were used to detect the expression levels of associated genes and proteins. IGF-1 (100 ng/ml) or VEGF $(25 \mathrm{ng} / \mathrm{ml})$ alone were revealed to be able to promote proliferation and migration of hCDPSCs; however, the combined use of IGF-1 and VEGF enhanced this effect when compared with the use of either agent in isolation. Alizarin red and ALP staining revealed that the use of either VEGF or IGF-1 alone did not result in any significant effects, whereas their use in combination promoted the osteogenic differentiation of hCDPSCs. In addition, the RT-qPCR and western blotting analyses revealed that the expression levels of Runt-related transcription factor 2 (RUNX2), bone sialoprotein (BSP) and ALP were increased upon combined treatment of the cells with VEGF and IGF-1. The expression levels of VEGF and plateletderived growth factor (PDGF) in hCDPSCs were enhanced upon treatment with either VEGF or IGF-1 in isolation, with greater effects observed when VEGF and IGF-1 were added in combination, indicating that VEGF and IGF-1 may exert a synergistic
\end{abstract}

Correspondence to: Professor Buling Wu, Department of Stomatology, Nanfang Hospital, Southern Medical University, 1838 North Guangzhou Avenue, Guangzhou, Guangdong 510515, P.R. China

E-mail: bulingwu@smu.edu.cn

Key words: human carious dental pulp stem cells, insulinlike growth factor-1, vascular endothelial growth factor, proliferation, differentiation, AKT pathway role in these events. Further experiments revealed that the combination of VEGF and IGF-1 led to an activation of the AKT signaling pathway. The proliferation and angiogenesis of hCDPSCs were also shown to be more effective compared with treatment with either VEGF or IGF-1 in isolation. Taken together, the present study has demonstrated that the combined use of VEGF and IGF-1 leads to an increase in the proliferation, migration, osteogenesis and angiogenesis of hCDPSCs and, furthermore, these signaling molecules may mediate their effects via activation of the AKT signaling pathway.

\section{Introduction}

Human dental pulp stem cells (hDPSCs) possess the ability to proliferate and to differentiate into odontoblasts, which enables them to participate in the reconstruction and repair of diseased pulp tissue (1-3). At present, dental caries, and the subsequent inflammation caused by dental pathogens, are common and nonnegligible clinical problems $(4,5)$. Human carious dental pulp stem cells (hCDPSCs) have attracted increasing levels of attention (6). Compared with normal hDPSCs, hCDPSCs have a number of advantages, such as a high proliferation rate and easy availability (6-9). hCDPSCs are considered to be ideal 'seed' cells in tissue engineering, although their characteristics have yet to be fully elucidated.

Growth factors (GFs) are types of polypeptide or glycoprotein that possess the biological properties of promoting cell proliferation, differentiation and locomotion (10-12). Among them, vascular endothelial growth factor (VEGF) and insulin-like growth factor-1 (IGF-1) are two important GFs with unique functions (13-15). VEGF has the capacity to trigger newly forming vessels in order to provide a blood supply for bone regeneration (16). IGF-1, a member of the insulin-like peptide family, is a ubiquitous and important peptide hormone and anti-apoptotic factor that exerts important roles in organ apoptosis and differentiation (17). It has been reported that IGF-1 may promote the proliferation of dental pulp stem cells (DPSCs) and periodontal ligament stem cells (PDLSCs), and induce their osteogenic/odontogenic differentiation $(18,19)$. It has been shown, that when either VEGF $(25 \mathrm{ng} / \mathrm{ml})$ or IGF-1 $(100 \mathrm{ng} / \mathrm{ml})$ was used, mesenchymal stem cells (MSCs) exhibited the strongest proliferative capacity (19-21). Furthermore, it was reported that the combined use of VEGF and IGF-1 enhanced osteogenic differentiation of 
periosteum-derived progenitor cells (PDPCs) and skin-derived MSCs (S-MSCs) (22). However, to the best of our knowledge, the effects of VEGF and IGF-1, either alone or in combination, on hCDPSCs has yet to be elucidated.

The phosphoinositide 3-kinase (PI3K)/Akt signaling pathway is an important signal transduction pathway, which tightly controls cell proliferation, migration, differentiation and self-renewal $(23,24)$. It has been reported that activation of the AKT signaling pathway led to an improvement in the proliferative and differentiation abilities of MSCs, thereby enhancing stemness (25).

In the present study, IGF-1 and VEGF, when applied either alone or in combination, were observed to exert an effect on the proliferation, migration and differentiation of hCDPSCs in vitro, and these effects were found to be associated with the AKT signaling pathway. These findings add to our knowledge of the cellular biological foundation for clinical application of hCDPSCs in the treatment of dental pulp disease.

\section{Materials and methods}

Isolation and culture of hCDPSCs. hCDPSCs were obtained from the carious pulp tissues, which were collected from teeth diagnosed with deep caries. The diagnosis of deep caries was determined by endodontic specialists on the basis of clinical assessment (26). A total of 20 patients (28-30 years of age; 10 male and 10 female patients) were informed about the nature of this research project, agreed to participate, and signed informed consent forms for scientific experiments involving tooth extraction in the Department of Stomatology of Nanfang Hospital, Southern Medical University (Guangzhou, China). The study protocol was performed according to a standard protocol approved by the Ethics Committee of the Southern Medical University. The pulp was carefully collected (1/3 of the apical pulp was discarded), cut into small pieces, and digested with collagenase $(3 \mathrm{mg} / \mathrm{ml}) /$ dispase enzyme $(4 \mathrm{mg} / \mathrm{ml}$; Sigma-Aldrich; Merck KGaA) for $30 \mathrm{~min}$. After 7-10 days, the primary cultured hCDPSCs were observed to be able to climb out along the edge of the tissue blocks. When the confluence of hCDPSCs reached $\sim 80 \%$, the cells were subcultured in new flasks. Cells were cultured with HyClone ${ }^{\mathrm{TM}} \alpha$-Minimal Essential Medium ( $\alpha$-MEM; Thermo Fisher Scientific, Inc.) containing $10 \%$ Gibco fetal bovine serum, $100 \mathrm{U} / \mathrm{ml}$ penicillin and $100 \mu \mathrm{g} / \mathrm{ml}$ streptomycin (all from Gibco; Thermo Fisher Scientific, Inc.). For all incubations in the methods section, unless specified otherwise, cells were cultured at $37^{\circ} \mathrm{C}$ in an atmosphere of $5 \% \mathrm{CO}_{2}$.

Phenotypic identification of hCDPSCs. Passage 3 hCDPSCs in the exponential growth phase were collected by $0.25 \%$ Trypsin and centrifuged at $1,500 \mathrm{x} \mathrm{g}$ for $1 \mathrm{~min}$ at $37^{\circ} \mathrm{C}$, and suspended in phosphate-buffered saline (PBS) to prepare a single cell suspension with a density of $1 \times 10 \% \mathrm{ml}$. The hCDPSCs were incubated with antihuman CD29PE (1:300, cat. no. 557332;), CD44PE (1:300, cat. no. 562818), CD45-PC5 (1:300, cat. no. 555484), CD90-PC5 (1:300, cat. no. 561972), CD105-PE (1:300, cat. no. 560839) and CD133-APC (1:300, cat. no. 566596; all from BD Biosciences, Franklin Lakes, NJ, USA) antibodies in different tubes at room temperature for 30 min, before washing three times with PBS and resuspending the cells in $300 \mu \mathrm{l}$ PBS. Fc blocking reagent was used to block the non-specific detection of the Fc component of all antibodies. Flow cytometry (BD Accuri C6, Becton Dickinson Biosciences) was used to detect the positive rate of stem cell surface markers. All data were analyzed by FlowJo Software (v10.0; FlowJo LLC).

Cell monoclonal assay and osteogenic induction. hCDPSCs $(2,000$ cells $)$ were inoculated into a $10 \mathrm{~cm}$ culture dish at $37^{\circ} \mathrm{C}$ in an atmosphere of $5 \% \mathrm{CO}_{2}$. After 14 days, hCDPSCs were fixed by $4 \%$ paraformaldehyde for $30 \mathrm{mins}$ and stained with Giemsa for 10 mins in $37^{\circ} \mathrm{C}$ Clones with $>50$ cells were counted as one unit.

Passage 3 hCDPSCs were cultured with osteogenic medium (10\% FBS, $10 \mathrm{mmol} / 1$ sodium $\beta$-glycerophosphate, $50 \mathrm{mg} / 1$ ascorbic acid and $0.1 \mu \mathrm{mol} / 1$ dexamethasone in $\alpha$-MEM). After 7 days of induction, hCDPSCs were fixed for 15 min with $4 \%$ paraformaldehyde, and subsequently incubated with ALP stain for $10 \mathrm{~min}$ at room temperature. After 21 days of induction, hCDPSCs were stained with Alizarin red for $10 \mathrm{~min}$ at room temperature following fixation with $4 \%$ paraformaldehyde. After a thorough rinse, the cells were observed and counted under a microscope a light microscope with x100 magnification.

Effect of VEGF and IGF-1 on proliferation and migration of hCDPSCs. Passage $3 \mathrm{hCDPSCs}$ in the exponential growth phase were collected by $0.25 \%$ Trypsin and centrifuged at $1,500 \mathrm{x} \mathrm{g}$ for $1 \mathrm{~min}$ at $37^{\circ} \mathrm{C}$, and inoculated (1,500 cells) into 96-well plates, and induced by the addition of no agent (blank control), IGF-1 (100 ng/ml), VEGF (25 ng/ml), or VEGF $(25 \mathrm{ng} / \mathrm{ml})+\mathrm{IGF}-1(100 \mathrm{ng} / \mathrm{ml})$, respectively. Cell proliferation was analyzed using a Cell Counting Kit-8 (CCK-8) assay (Beyotime Institute of Biotechnology). According to the manufacturer's protocol, on days 1, 3, 5, 7 and 9, the absorbance at $490 \mathrm{~nm}$ wavelength was measured following incubation with CCK8 reagent for $3 \mathrm{~h}$. Similarly, after the cells were cultured in the different experimental group set-ups, migration assay was performed in a Transwell chamber. Serum-free medium was added in the upper chamber, and $5 \times 10^{4}$ cells were inoculated in each well. $10 \%$ FBS medium was added into the lower chamber. The cells were incubated at $37^{\circ} \mathrm{C}$ in an atmosphere of $5 \% \mathrm{CO}_{2}$ for $24 \mathrm{~h}$. Subsequently, the supernatant was discarded, the cells were fixed in $4 \%$ paraformaldehyde for $30 \mathrm{~min}$ and stained by $0.1 \%$ crystal violet in $37^{\circ} \mathrm{C}$.

Reverse transcription-quantitative polymerase chain reaction ( $R T$ - $q P C R)$. Total RNA was extracted from cells in the different groups using TRIzol ${ }^{\circledR}$, and total RNA was reverse-transcribed into cDNA according to the instructions of the RT kit employed (Qiagen $\mathrm{GmbH}$ ). The content of RNA was quantified (Nanodrop; Thermo Fisher Scientific, Inc.). SYBR Green was purchased from Qiagen $\mathrm{GmbH}$ and the sequences of the RT-PCR primers (Thermo Fisher Scientific, Inc.) are given in Table I. GAPDH was used as reference gene. The following thermocycling conditions were used: $95^{\circ} \mathrm{C}$ for $20 \mathrm{sec}$, annealing at $60^{\circ} \mathrm{C}$ for $30 \mathrm{sec}$, and extension at $72^{\circ} \mathrm{C}$ for $30 \mathrm{sec}$ (40 cycles). Each assay was performed in triplicate, and quantification was performed using the $2^{-\Delta \Delta \mathrm{Cq}}$ method (27). 
Table I. Primers used for reverse transcription-quantitative PCR.

\begin{tabular}{ll}
\hline Gene & \multicolumn{1}{c}{ Sequence } \\
\hline RUNX2 & F: 5'TGGTTACTGTCATGGCGGGTA3' \\
& R: 5'TCTCAGATCGTTGAACCTTGCTA3' \\
BSP & F: 5'CACTGGAGCCAATGCAGAAGA3' \\
& R: 5'TGGTGGGGTTGTAGGTTCAAA3' \\
ALP & F:5'GAGATGTTGTCCTGACACTTGTG3' \\
& R: 5'AGGCTTCCTCCTTGTTGGGT3' \\
VEGF & F: 5'AGGGCAGAATCATCACGAAGT3' \\
& R: 5'AGGGTCTCGATTGGATGGCA3' \\
PDGF & F: 5'TGGCAGTACCCCATGTCTGAA3' \\
& R: 5'CCAAGACCGTCACAAAAAGGC3' \\
GAPDH & F: 5'ACAACTTTGGTATCGTGGAAGG3' \\
& R: 5'GCCATCACGCCACAGTTTC3'
\end{tabular}

RUNX2, Runt-related transcription factor 2; BSP, bone sialoprotein; ALP, alkaline phosphatase; VEGF, vascular endothelial growth factor; PDGF, platelet-derived growth factor; GAPDH, glyceraldehyde-3-phosphate dehydrogenase.

Western blot analysis. Passage 3 hCDPSCs from the different groups were collected by $0.25 \%$ Trypsin and centrifuged at $1,500 \mathrm{xg}$ for $1 \mathrm{~min}$ at $37^{\circ} \mathrm{C}$ and washed twice with ice-cold PBS, and subsequently the total protein was extracted using RIPA buffer (Beyotime Institute of Biotechnology) (28). Aliquots of $40 \mu \mathrm{g}$ protein from each sample were subjected to SDSPAGE ( $10 \%$ gels), and the proteins were subsequently transferred on to PVDF membranes (Life Sciences, Ann Arbor, MI, USA). The membranes were subsequently blocked with TBST containing $5 \%$ defatted milk powder at room temperature for $1 \mathrm{~h}$, and then incubated overnight with primary antibodies against RUNX2 (1:500, cat. no. 12556), BSP (1:500, cat. no. 5468S), ALP (1:500, cat. no. 8681), VEGF (1:500, cat. no. 2463), PDGF (1:500, cat. no. 3169), AKT (1:500, cat. no. 4685), phosphorylated (p)-AKT (1:500, cat. no. 9614) and cyclin D1 (1:500, cat. no. 2978; all antibodies from Cell Signaling Technology, Danvers, MA, USA) at $4^{\circ} \mathrm{C}$ overnight. The membranes were subsequently washed with PBS three times. Peroxidase-conjugated goat anti-rabbit or goat anti-mouse was used as the secondary antibody (cat. no. 4414 or $4410 \mathrm{~S}$, respectively; 1:1,000 dilution; Cell Signaling Technology), and the membranes were incubated with secondary antibody for $1 \mathrm{~h}$ at room temperature, prior to subsequent exposure to an Odyssey 2-colour infrared laser imaging system (LI-COR Biosciences, Lincoln, NE, USA). The semi-quantitative results for each western blot were measured using the Image-Pro Plus 6.0 program (Media Cybernetics, Inc., Rockville, MD, USA).

In vitro Matrigel ${ }^{T M}$ tube formation assay. Passage 3 hCDPSCs in the exponential growth phase were collected by $0.25 \%$ Trypsin and centrifuged at $1,500 \mathrm{xg}$ for $1 \mathrm{~min}$ at $37^{\circ} \mathrm{C}$, and cell suspensions $\left(1 \times 10^{6}\right.$ cells) were prepared. Matrigel ${ }^{\mathrm{TM}}$ was coated onto 12-well plates according to the manufacturer's protocol $(29,30)$. The cells were gently mixed, inoculated on to the aggregated Matrigel ${ }^{\mathrm{TM}}$, and subsequently were incubated in $37^{\circ} \mathrm{C}$ in an atmosphere of $5 \% \mathrm{CO}_{2}$ for $7 \mathrm{~h}$. The condition of the vessels was observed under a light microscope, and the density of vessels was determined using Image-Pro Plus 6.0 program (Media Cybernetics, Inc.).

Statistical analysis. SPSS 17.0 was used for statistical analysis. Data are expressed as the mean \pm standard deviation. Comparisons among groups was performed using one-way ANOVA, and post-hoc comparisons were performed by the least significant difference (LSD) $t$ test. $\mathrm{P}<0.05$ was considered to indicate a statistically significant difference.

\section{Results}

Identification of hCDPSCs. Passage 3 hCDPSCs were collected and cultured for stem cell identification. The cells exhibited the property of monoclonal formation, as well as osteogenic differentiation and mineralization (Fig. 1A-C). The flow cytometry experiments revealed positive expression of CD29, CD44, CD90 and CD105, and negative expression of CD45 and CD133 (Fig. 1D). According to the criteria for defining multipotent MSCs, the isolated human carious dental pulp cells were identified as MSCs.

Effects of VEGF and IGF-1 on proliferation and migration of hCDPSCs. CCK8 assay revealed that the proliferation of hCDPSCs could be stimulated by adding either VEGF or IGF-1 alone, whereas the addition of VEGF and IGF-1 in combination led to a further enhancement in the proliferation rate of the hCDPSCs (Fig. 2A).

Transwell assay was subsequently used to detect the migratory ability of hCDPSCs upon treatment with VEGF and IGF-1, either separately or in combination. The results demonstrated that there was a synergistic effect of VEGF/IGF-1 treatment on cell migration, and the effect of combined treatment was greater than that of either of these two GFs when added in isolation (Fig. 2B).

Effects of VEGF and IGF-1 on osteogenic differentiation of hCDPSCs in vitro. For these experiments, five experimental groups were established: Blank control (Con), ordinary osteogenic medium (OM), osteogenic medium plus VEGF (VEGF + $\mathrm{OM})$, osteogenic medium plus IGF-1 (IGF-1 + OM), and osteogenic medium plus VEGF and IGF-1 (VEGF + IGF-1 + OM). Alizarin red staining and ALP staining revealed that, compared with the OM group, the osteogenic ability of the VEGF + IGF-1 + OM group revealed a significant enhancement in the staining intensity (Fig. 3A-C). The corresponding RT-qPCR and western blotting results revealed that the mRNA and protein expression levels of the osteogenesisassociated genes (i.e., Runx2, BSP and ALP) increased significantly in the VEGF + OM and IGF-1 + OM groups, whereas the largest increase was observed for the VEGF + IGF-1 + OM group (Fig. 4A-C).

Effects of VEGF and IGF-1 on angiogenic differentiation of $h C D P S C s$ in vitro. Tube formation assays revealed that the number of tubules could be increased by adding either VEGF or IGF-1 alone, whereas the addition of VEGF and IGF in combination led to a further increase in the angiogenic ability of hCDPSCs (Fig. 5B and C). The RT-qPCR and 

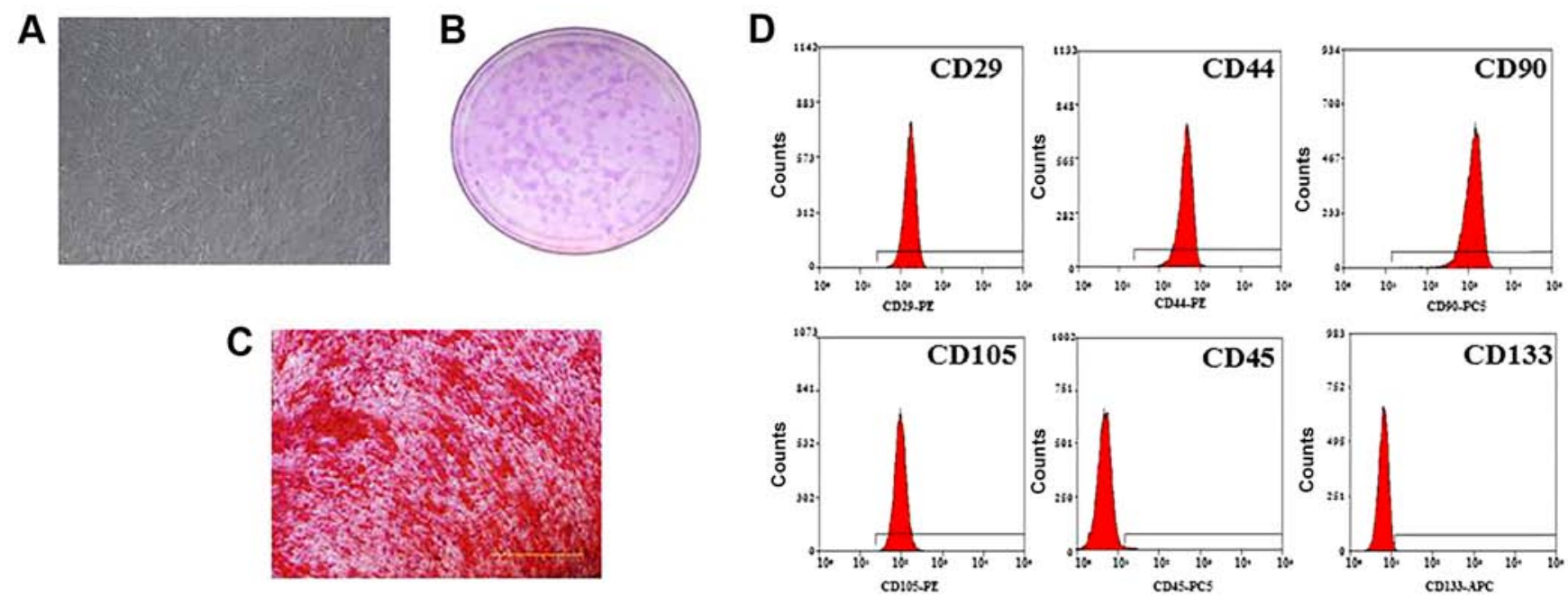

Figure 1. Identification of hCDPSCs. The (A) morphology, (B) colony-forming ability, and (C) osteogenic differentiation of hCDPSCs are shown. (D) Flow cytometric analysis confirmed that the hCDPSCs positively expressed CD29, CD44, CD90 and CD105, whereas CD45 and CD133 were negatively expressed. Scale bar, $200 \mu \mathrm{m}$. hCDPSCs, human carious dental pulp stem cells.

A

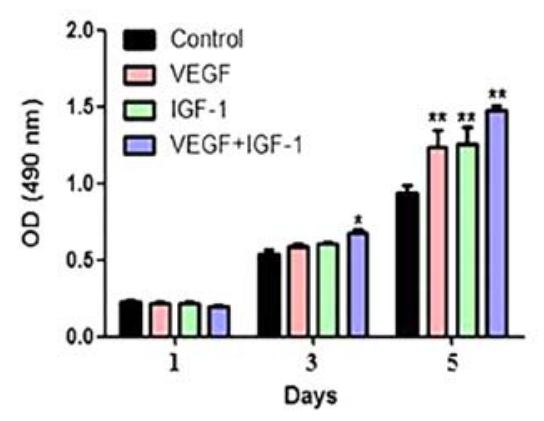

B

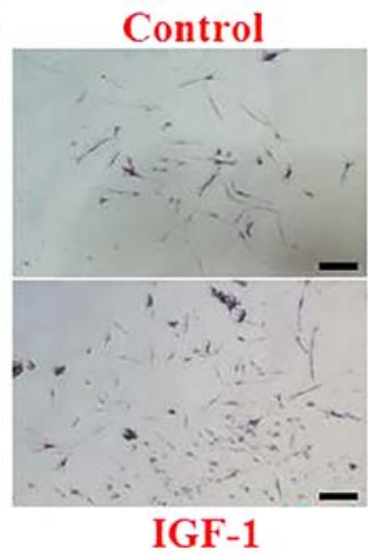

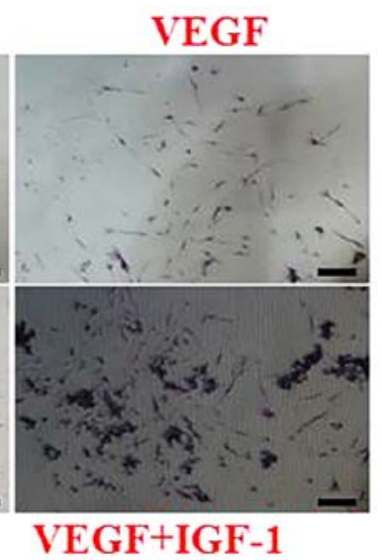

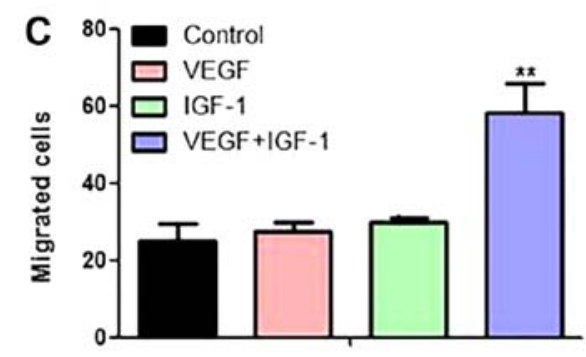

Figure 2. Proliferation and migration of hCDPSCs upon treatment with VEGF and IGF-1. (A) The CCK8 assay revealed the proliferation rates of hCDPSCs in the different groups. (B) Migration of hCDPSCs in the different groups was tested by Transwell assay, and (C) the numbers of migrated cells were calculated. Scale bar, $200 \mu \mathrm{m} .{ }^{*} \mathrm{P}<0.05,{ }^{* *} \mathrm{P}<0.01$ vs. control. hCDPSCs, human carious dental pulp stem cells; IGF-1, insulinlike growth factor 1; VEGF, vascular endothelial growth factor; CCK8, Cell Counting kit-8.

western blotting results exhibited a similar trend to that of the osteogenesis-induction assay: The expression of vessel formation-associated genes and proteins (i.e., VEGF and PDGF) increased significantly in the OM + VEGF and OM +I GF-1 groups, whereas the VEGF + IGF-1 + OM group exhibited the greatest enhancement (Fig. 5A, D and E).

Effect of VEGF and IGF-1 on the AKT signaling pathway in hCDPSCs in vitro. Compared with the control group, the expression levels of p-AKT and cyclin D1 were increased upon addition of either VEGF or IGF-1 alone, whereas the expression of these two proteins was further increased by adding VEGF and IGF-1 in combination to activate the AKT signaling pathway of the hCDPSCs (Fig. 6). These results demonstrated that the combination of VEGF and IGF-1 elicited a synergistic effect (Fig. 6).

\section{Discussion}

Currently, hDPSCs are commonly used as 'seed' cells in bone tissue engineering due to their high self-renewal capacity and 
A
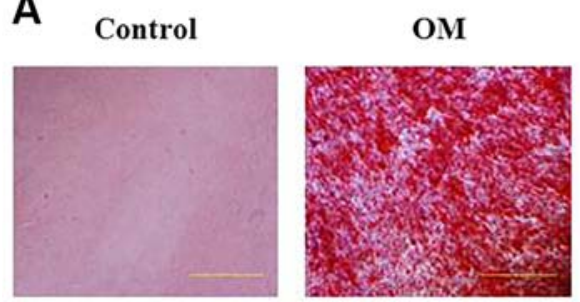

VEGF+OM

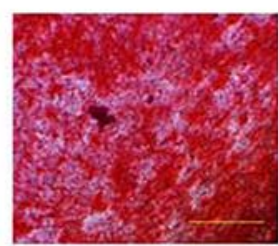

IGF-1+OM

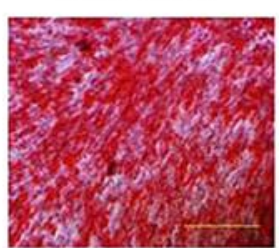

VEGF+IGF-

$1+\mathrm{OM}$

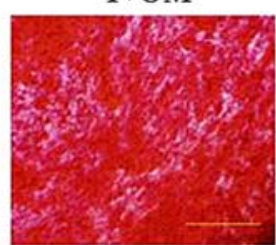

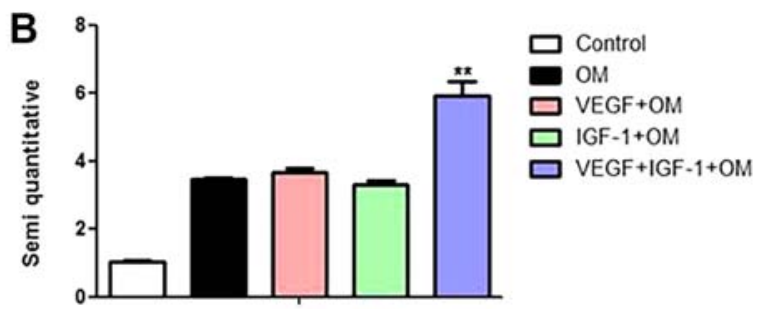

C Control

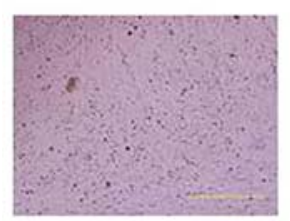

OM

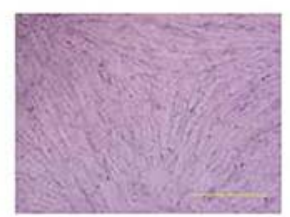

$\mathrm{VEGF}+\mathrm{OM}$

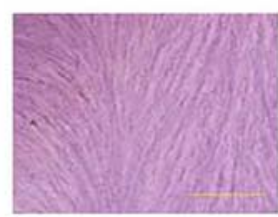

IGF-1+OM

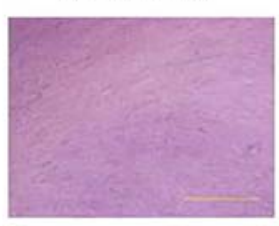

VEGF+IGF $1+\mathrm{OM}$

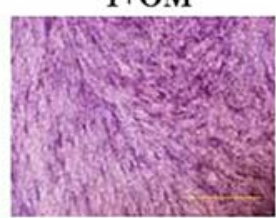

Figure 3. Osteogenic differentiation of hCDPSCs upon treatment with VEGF and IGF-1. (A) The osteogenic differentiation capability of hCDPSCs was examined using Alizarin red staining, with (B) subsequent quantification of the results. ${ }^{* *} \mathrm{P}<0.01$ vs. control. (C) The results from ALP staining are shown. For the description of the experimental groups (Control, OM, VEGF+OM, IGF-1+OM and VEGF+IGF-1+OM), see the Results section. Scale bar, $200 \mu \mathrm{m}$. hCDPSCs, human carious dental pulp stem cells; IGF-1, insulinlike growth factor 1; VEGF, vascular endothelial growth factor; ALP, alkaline phosphatase.
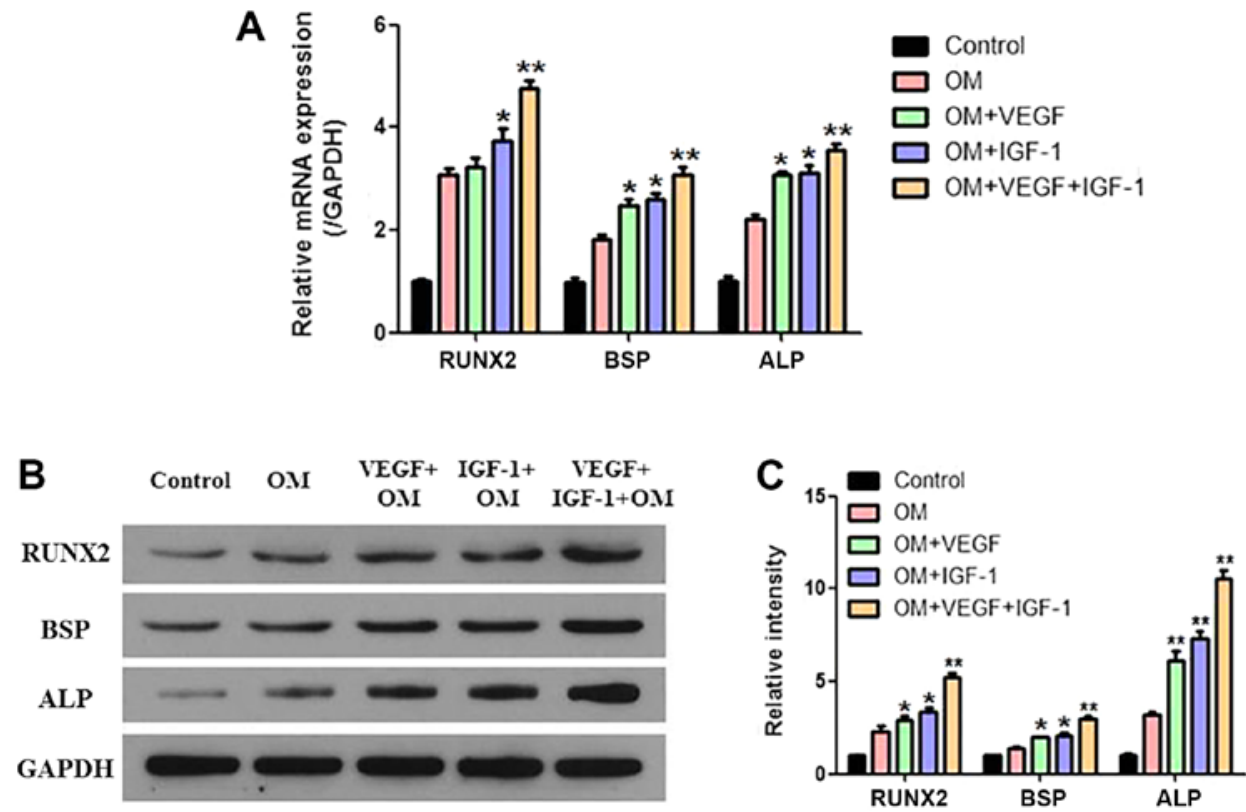

Figure 4. Analysis of the osteogenesis-associated genes and proteins of hCDPSCs upon treatment with VEGF and IGF-1. (A) The mRNA expression levels of the RUNX2, BSP and ALP genes were examined by RT-qPCR. (B) The protein expression levels of RUNX2, BSP and ALP were examined by western blotting, and $(\mathrm{C})$ the relative protein expression levels were normalized against GAPDH. ${ }^{*} \mathrm{P}<0.05,{ }^{* *} \mathrm{P}<0.01$ vs. control. For the description of the experimental groups (Control, OM, VEGF+OM, IGF-1+OM and VEGF+IGF-1+OM), see the Results section. hCDPSCs, human carious dental pulp stem cells; IGF-1, insulin-like growth factor 1; VEGF, vascular endothelial growth factor; RUNX2, Runt-related transcription factor 2; BSP, bone sialoprotein; ALP, alkaline phosphatase; GAPDH, glyceraldehyde-3-phosphate dehydrogenase.

stemness $(3,31)$. hCDPSCs are a unique type of dental stem cell, since they are derived from the pulp in deep carious teeth $(7,8)$. This special environmental stimulus enables the stronger proliferation and osteogenic differentiation capability of hCDPSCs, and therefore they are now recognized as a potential source for regenerative medicine $(7,8)$. 
A
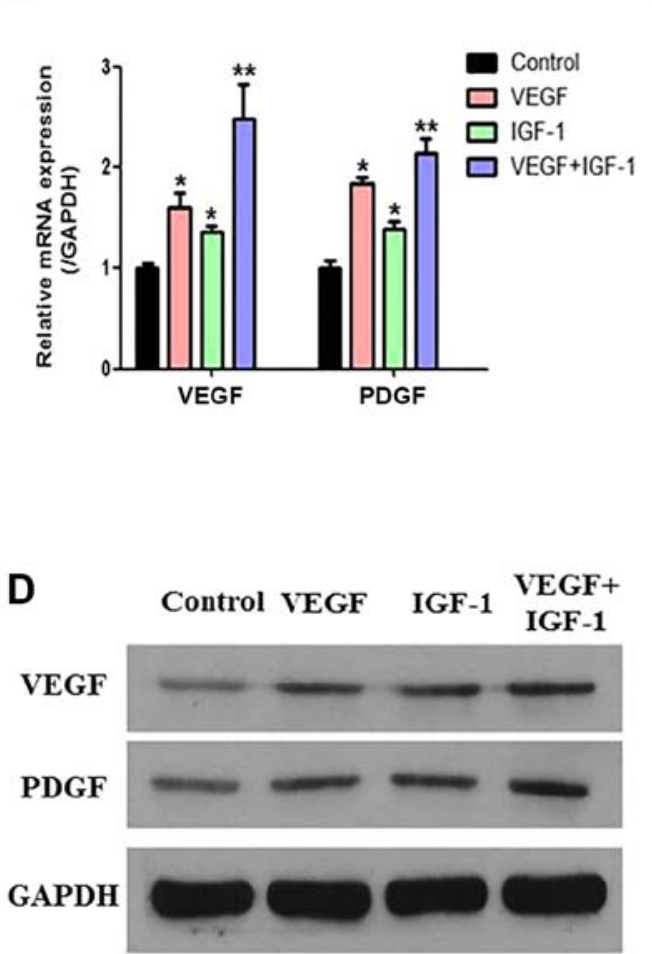

B
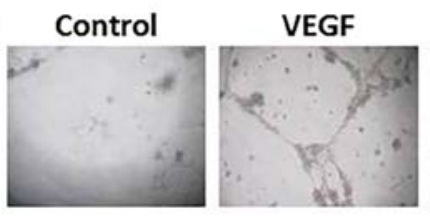

IGF-1 VEGF+IGF-1

C

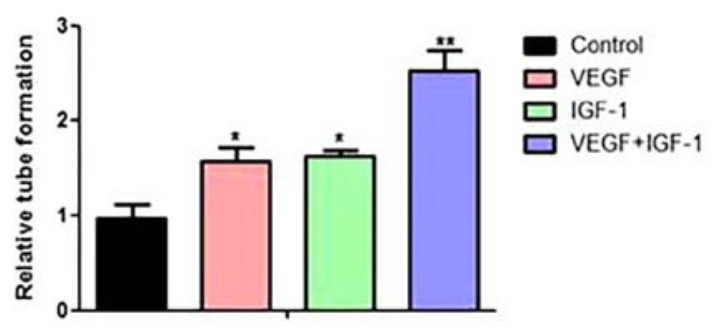

E

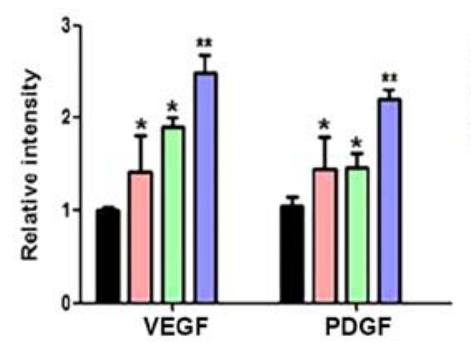

Figure 5. Angiogenic differentiation of hCDPSCs upon treatment with VEGF and IGF-1. (A) mRNA expression levels of VEGF and PDGF were examined by RT-qPCR. (B and C) Tube-formation ability was examined in the different groups. Magnification, x200. (D) The protein expression levels of VEGF and PDGF were detected by western blotting, and (E) the relative protein expression level was normalized against GAPDH. ${ }^{*} \mathrm{P}<0.05$, ${ }^{* * *} \mathrm{P}<0.01 \mathrm{vs}$. control. For the description of the experimental groups (Control, VEGF, IGF-1 and VEGF+IGF-1), see the Results section. hCDPSCs, human carious dental pulp stem cells; IGF-1, insulin-like growth factor 1; VEGF, vascular endothelial growth factor; PDGF, platelet-derived growth factor; GAPDH, glyceraldehyde-3-phosphate dehydrogenase.
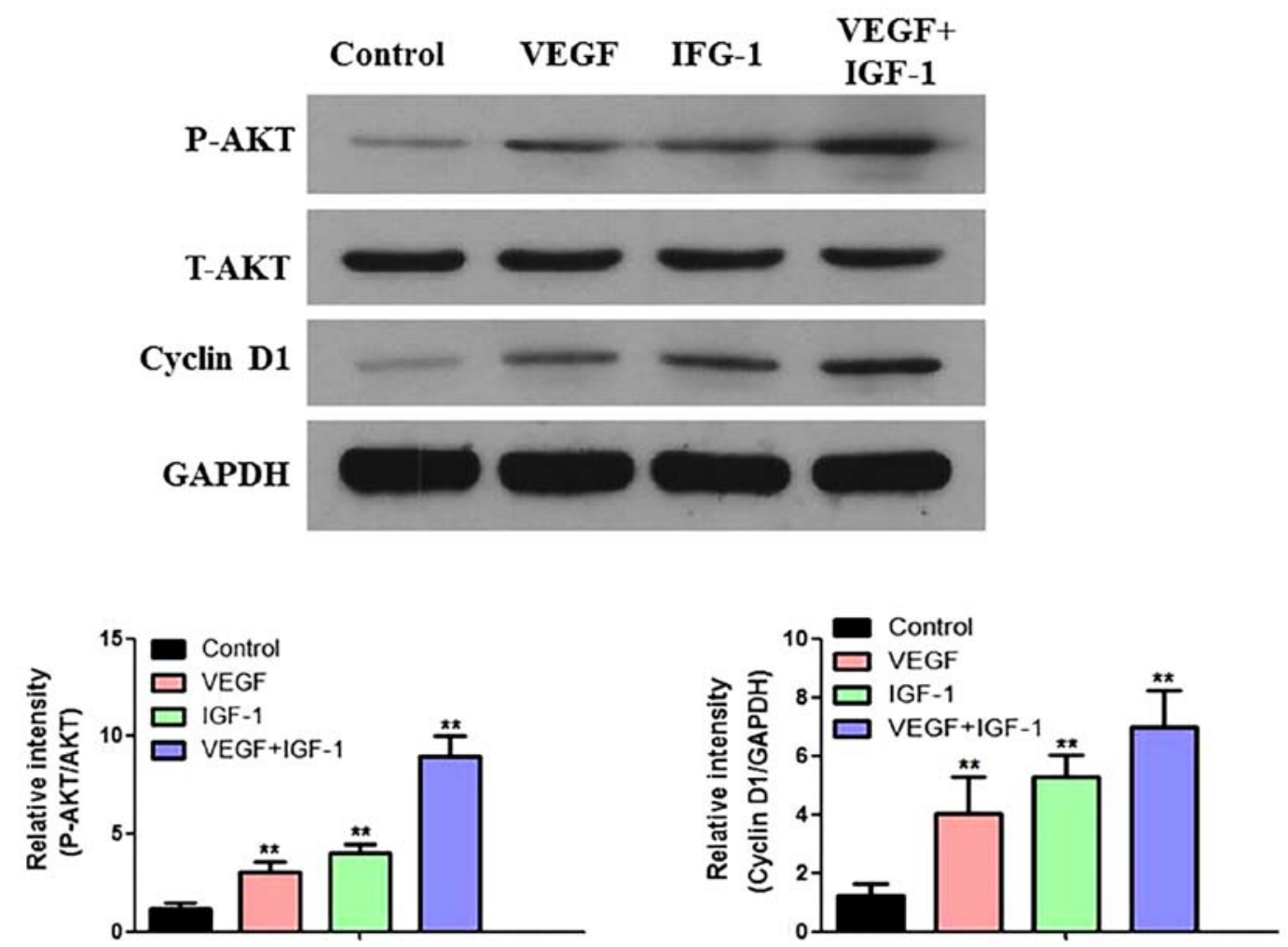

Figure 6. Effect of AKT signaling pathway in hCDPSCs upon treatment with VEGF and IGF-1. The protein expression levels of P-AKT, T-AKT and cyclin D1 were detected by western blotting, and the relative protein expression levels were calculated. ${ }^{* *} \mathrm{P}<0.01$ vs. control. For the description of the experimental groups (Control, VEGF, IGF-1 and VEGF+IGF-1), see the Results section. hCDPSCs, human carious dental pulp stem cells; IGF-1, insulin-like growth factor 1; VEGF, vascular endothelial growth factor; P-AKT, phosphorylated AKT; T-AKT, total AKT. 
GFs are critical in tissue regeneration, as they have important roles in regulating cell functions $(32,33)$. Previous studies have reported that the combination of IGF-1 and other GFs (such as PDGF-B or BMP-2) may affect cell proliferation, promote osteogenesis, and help the reconstruction of toothsupporting tissues (34-36). IGF-1 has been identified to stimulate osteogenic differentiation via the mitogen-activated protein kinase signaling pathway, suggesting that IGF-1 has a role in regeneration of the periodontal tissue (37). VEGF has the potential of triggering neovascularization, which is able to regulate both normal and pathological conditions, and provides blood supply for tissue regeneration $(38,39)$. Angiogenesis is essential for bone reconstruction, as new bone formation relies on a suitable blood supply to provide cells and nutrients (40). Therefore, the selection of suitable GFs has become an important research focus in tissueengineering therapy.

In the present study, the CCK8 assay results demonstrated that the combination of VEGF and IGF-1 exerted a synergistic effect on proliferation. Transwell assay revealed that the migratory ability of hCDPSCs upon combined treatment of VEGF and IGF-1 was markedly stronger compared with that of treating with VEGF or IGF-1 alone. This finding indicated that there was a synergistic effect resulting from treating the cells with VEGF and IGF-1 in combination. It has been previously shown that, when either VEGF $(25 \mathrm{ng} / \mathrm{ml})$ or IGF-I (100 ng/ml) were used, MSCs exhibited the strongest proliferative capacity (19-21). Furthermore, it was reported that the combined use of VEGF and IGF-1 enhanced osteogenic PDPCs and S-MSCs (22). Therefore, based on the findings of these previous studies, a combination of IGF-1 (100 ng/ml) and VEGF $(25 \mathrm{ng} / \mathrm{ml})$ was used in the present study to investigate the effects of IGF-1 and VEGF on proliferation, migration, osteogenesis and vascularization of hCDPSCs.

RUNX2 is a highly conserved transcription factor, known as the most important regulator in osteoblast and odontoblast differentiation (41). Runx 2 activates boneassociated genes and promotes mineralization in the early stage of osteoblast differentiation $(41,42)$. ALP is the enzyme that is predominantly involved in both bone and tooth mineralization, and elevated levels of ALP may be considered as an early marker of odontoblast differentiation and dentin formation (43). BSP is an important product during osteogenic differentiation, which may clearly indicate the level of ALP (44). The results of RT-PCR and western blotting in the present study demonstrated that VEGF and IGF-1 exerted a synergistic effect on bone formation, which was more effective than using either of the drugs alone. It has been reported that the combined use of VEGF and IGF-1 could induce the differentiation of S-MSCs into osteoblasts, and that the IGF-1 and insulin signaling pathways may function as important mediators in terms of Runx2 activity (45).

The results from the alizarin red and ALP staining experiments in the present study revealed that the combined use of VEGF and IGF-1 could promote the osteogenesis of hCDPSCs. VEGF has been widely studied as a major regulator in angiogenesisassociated processes, in which the molecule exerts both direct and indirect functions (46). VEGF stimulates cell proliferation and migration, whereas on the other hand, it increases the permeability of blood vessels and allows plasma proteins to leak out of blood vessels, a process that serves an important role in remodeling the extracellular matrix to adapt to angiogenesis $(46,47)$. The addition of VEGF during the early stage of bone repair may lead to an improvement in the blood supply of the microenvironment and the formation of new bone (47). The results of the present study showed that the single application of either VEGF or IGF-1 was sufficient to promote vascular differentiation of hCDPSCs, whereas the combined use of VEGF and IGF-1 elicited a synergistic effect.

The PI3K/Akt pathway is a tyrosine kinase receptor-mediated signaling system, which exists widely in various types of cell (23). It is an important signal-transduction pathway involved in the regulation of cell growth, proliferation and differentiation (24). Activated Akt can induce changes in a series of downstream factors [including mammalian target of rapamycin, (mTOR), glycogen synthase kinase 3 (GSK3), Bax, NF- $\kappa \mathrm{B}$, caspases, etc.] and participate in the regulation of cell growth, differentiation, division and migration (24). The phosphorylation level of Akt may also reflect the activity of whole signaling pathway (48). It has been shown that IGF-1 promotes the vascular differentiation of adipose-derived stem cells and endothelial cells via the PI3K/AKT signaling pathway (48). The activation of autophagic activity is able to enhance the osteogenic differentiation of hBMSCs, thereby providing a novel therapeutic target for osteoporosis treatment (49). Therefore, it is possible to surmise that activation of the PI3K-AKT pathway may give rise to the observed effects associated with the mTOR pathway and autophagy activity during the osteogenic differentiation of hCDPSCs.

In conclusion, the present study has identified that the AKT signaling pathway may be activated by the use of VEGF or IGF-1 alone, whereas the AKT signaling pathway can be further activated by the combined use of VEGF and IGF-1. This suggests that the combined use of VEGF and IGF-1 may have the additive effects through AKT signaling pathway.

\section{Acknowledgements}

Not applicable.

\section{Funding}

This study was supported by grant from the National Natural Science Foundation of China (grant no. 8187041227).

\section{Availability of data and materials}

The datasets used and/or analyzed during the current study are available from the corresponding author on reasonable request.

\section{Authors' contributions}

BW and WL designed the study. WL, WX and JL guided the experiments. WL, YC and YP performed the experiments. WL and WX collected and processed the clinical data. JL, YC and YP analyzed and interpreted the patient data. BW and WL wrote the paper, and BW reviewed and edited the manuscript. All authors read and approved the final manuscript. 


\section{Ethics approval and consent to participate}

The study protocol was performed according to a standard protocol approved by the Ethics Committee of the Southern Medical University. All patients signed written informed consent forms.

\section{Patient consent for publication}

Not applicable.

\section{Competing interests}

The authors declare that they have no competing interests.

\section{References}

1. Xuan K, Li B, Guo H, Sun W, Kou X, He X, Zhang Y, Sun J, Liu A, Liao L, et al: Deciduous autologous tooth stem cells regenerate dental pulp after implantation into injured teeth. Sci Transl Med 10: eaaf3227, 2018.

2. Xia Y, Chen H, Zhang F, Bao C, Weir MD, Reynolds MA, Ma J, $\mathrm{Gu} \mathrm{N}$ and $\mathrm{Xu} \mathrm{HHK}$ : Gold nanoparticles in injectable calcium phosphate cement enhance osteogenic differentiation of human dental pulp stem cells. Nanomedicine 14: 35-45, 2018.

3. Martin-Del-Campo M, Rosales-Ibanez R, Alvarado K, Sampedro JG, Garcia-Sepulveda CA, Deb S, San Román J and Rojo L: Strontium folate loaded biohybrid scaffolds seeded with dental pulp stem cells induce in vivo bone regeneration in critical sized defects. Biomater Sci 4: 1596-1604, 2016.

4. Farges JC, Alliot-Licht B, Renard E, Ducret M, Gaudin A, Smith AJ and Cooper PR: Dental pulp defence and repair mechanisms in dental caries. Mediators Inflamm 2015: 230251, 2015.

5. Fernandez MR, Goettems ML, Demarco FF and Correa MB: Is obesity associated to dental caries in Brazilian schoolchildren? Braz Oral Res 31: e83, 2017.

6. Louvrier A, Euvrard E, Nicod L, Rolin G, Gindraux F, Pazart L, Houdayer C, Risold PY, Meyer F and Meyer C: Odontoblastic differentiation of dental pulp stem cells from healthy and carious teeth on an original PCL-based 3D scaffold. Int Endod J 51 (Suppl 4): e252-e263, 2018.

7. Gnanasegaran N, Govindasamy V and Abu Kasim NH: Differentiation of stem cells derived from carious teeth into dopaminergic-like cells. Int Endod J 49: 937-949, 2016.

8. Werle SB, Lindemann D, Steffens D, Demarco FF, de Araujo FB, Pranke $P$ and Casagrande L: Carious deciduous teeth are a potential source for dental pulp stem cells. Clin Oral Investig 20: 75-81, 2016.

9. Ma D, Gao J, Yue J, Yan W, Fang F and Wu B: Changes in proliferation and osteogenic differentiation of stem cells from deep caries in vitro, J Endod 38: 796-802, 2012.

10. Sinclair KL, Mafi P, Mafi R and Khan WS: The use of growth factors and mesenchymal stem cells in orthopaedics: In particular, their use in Fractures and Non-Unions: A systematic review. Curr Stem Cell Res Ther 12: 312-325, 2017.

11. Augustyniak E, Trzeciak T, Richter M, Kaczmarczyk J and Suchorska W: The role of growth factors in stem cell-directed chondrogenesis: A real hope for damaged cartilage regeneration. Int Orthop 39: 995-1003, 2015.

12. Hankenson KD, Gagne K and Shaughnessy M: Extracellular signaling molecules to promote fracture healing and bone regeneration. Adv Drug Deliv Rev 94: 3-12, 2015.

13. Kim Y and Liu JC: Protein-engineered microenvironments can promote endothelial differentiation of human mesenchymal stem cells in the absence of exogenous growth factors. Biomater Sci 4: 1761-1772, 2016

14. Trosan P, Javorkova E, Zajicova A, Hajkova M, Hermankova B, Kossl J, Krulova M and Holan V: The Supportive Role of insulin-like growth factor-I in the differentiation of murine mesenchymal stem cells into corneal-like cells. Stem Cells Dev 25: 874-881, 2016.

15. Chen CY, Tseng KY, Lai YL, Chen YS, Lin FH and Lin S: Overexpression of insulin-like growth factor 1 enhanced the osteogenic capability of aging bone marrow mesenchymal stem cells. Theranostics 7: 1598-1611, 2017.
16. Shi C, Zhao Y, Yang Y, Chen C, Hou X, Shao J, Yao H, Li Q, $\mathrm{Xia} Y$ and Dai J: Collagen-binding VEGF targeting the cardiac extracellular matrix promotes recovery in porcine chronic myocardial infarction. Biomater Sci 6: 356-363, 2018.

17. Rybalko VY, Pham CB, Hsieh PL, Hammers DW, Merscham-Banda M, Suggs LJ and Farrar RP: Controlled delivery of SDF-1 $\alpha$ and IGF-1: CXCR4(+) cell recruitment and functional skeletal muscle recovery. Biomater Sci 3: 1475-1486, 2015.

18. Feng X, Huang D, Lu X, Feng G, Xing J, Lu J, Xu K, Xia W, Meng Y, Tao T, et al: Insulin-like growth factor 1 can promote proliferation and osteogenic differentiation of human dental pulp stem cells via mTOR pathway. Dev Growth Differ 56: 615-624, 2014.

19. Yu Y, Mu J, Fan Z, Lei G, Yan M, Wang S, Tang C, Wang Z, $\mathrm{Yu} \mathrm{J}$ and Zhang G: Insulin-like growth factor 1 enhances the proliferation and osteogenic differentiation of human periodontal ligament stem cells via ERK and JNK MAPK pathways. Histochem Cell Biol 137: 513-525, 2012.

20. Bai Y, Li P, Yin G, Huang Z, Liao X, Chen X and Yao Y: BMP-2, VEGF and bFGF synergistically promote the osteogenic differentiation of rat bone marrow-derived mesenchymal stem cells. Biotechnol Lett 35: 301-308, 2013.

21. Lee JH, Um S, Jang JH and Seo BM: Effects of VEGF and FGF-2 on proliferation and differentiation of human periodontal ligament stem cells. Cell Tissue Res 348: 475-484, 2012.

22. Dicarlo M, Bianchi N, Ferretti C, Orciani M, Di Primio R and Mattioli-Belmonte M: Evidence supporting a paracrine effect of IGF-1/VEGF on human mesenchymal stromal cell commitment. Cells Tissues Organs 201: 333-341, 2016.

23. Zhang J, Liu X, Li H, Chen C, Hu B, Niu X, Li Q, Zhao B, Xie Z and Wang Y: Exosomes/tricalcium phosphate combination scaffolds can enhance bone regeneration by activating the PI3K/Akt signaling pathway. Stem Cell Res Ther 7: 136, 2016.

24. Wu X, Zheng S, Ye Y, Wu Y, Lin K and Su J: Enhanced osteogenic differentiation and bone regeneration of poly(lactic-co-glycolic acid) by graphene via activation of PI3K/Akt/GSK-3 $\beta / \beta$-catenin signal circuit. Biomater Sci 6: 1147-1158, 2018.

25. Iyer S, Viernes DR, Chisholm JD, Margulies BS and Kerr WG: SHIP1 regulates MSC numbers and their osteolineage commitment by limiting induction of the PI3K/Akt//catenin/Id2 axis. Stem Cells Dev 23: 2336-2351, 2014.

26. Ma D, Cui L, Gao J, Yan W, Liu Y, Xu S and Wu B: Proteomic analysis of mesenchymal stem cells from normal and deep carious dental pulp. PLoS One 9: e97026, 2014.

27. Livak KJ and Schmittgen TD: Analysis of relative gene expression data using real-time quantitative PCR and the 2(-Delta Delta C(T)) method. Methods 25: 402-408, 2001.

28. Pan Y, Chen J, Yu Y, Dai K, Wang J and Liu C: Enhancement of BMP-2-mediated angiogenesis and osteogenesis by 2-N,6-O-sulfated chitosan in bone regeneration. Biomater Sci 6: 431-439, 2018.

29. Gangadaran P, Rajendran RL, Lee HW, Kalimuthu S, Hong CM, Jeong SY, Lee SW, Lee J and Ahn BC: Extracellular vesicles from mesenchymal stem cells activates VEGF receptors and accelerates recovery of hindlimb ischemia. J Control Release 264: 112-126, 2017.

30. Thekkeparambil Chandrabose S, Sriram S, Subramanian S, Cheng S, Ong WK, Rozen S, Kasim NHA and Sugii S: Amenable epigenetic traits of dental pulp stem cells underlie high capability of xeno-free episomal reprogramming. Stem Cell Res Ther 9: 68, 2018.

31. Schreier C, Rothmiller S, Scherer MA, Rummel C, Steinritz D, Thiermann $\mathrm{H}$ and Schmidt A: Mobilization of human mesenchymal stem cells through different cytokines and growth factors after their immobilization by sulfur mustard. Toxicol Lett 293: 105-111, 2018.

32. Alarcin E, Lee TY, Karuthedom S, Mohammadi M, Brennan MA, Lee DH, Marrella A, Zhang J, Syla D, Zhang YS, et al: Injectable shear-thinning hydrogels for delivering osteogenic and angiogenic cells and growth factors. Biomater Sci 6: 1604-1615, 2018

33. Moeller M, Pink C, Endlich N, Endlich K, Grabe HJ, Volzke H, Dörr M, Nauck M, Lerch MM, Köhling R, et al: Mortality is associated with inflammation, anemia, specific diseases and treatments, and molecular markers. PLoS One 12: e175909, 2017.

34. Schminke B, Vom Orde F, Gruber R, Schliephake H, Burgers R and Miosge N: The pathology of bone tissue during peri-implantitis. J Dent Res 94: 354-361, 2015. 
35. Chang PC, Cirelli JA, Jin Q, Seol YJ, Sugai JV, D'Silva NJ, Danciu TE, Chandler LA, Sosnowski BA and Giannobile WV: Adenovirus encoding human platelet-derived growth factor-B delivered to alveolar bone defects exhibits safety and biodistribution profiles favorable for clinical use. Hum Gene Ther 20 486-496, 2009.

36. Zhang C, Hong FF, Wang CC, Li L, Chen JL, Liu F, Quan RF and Wang JF: TRIB3 inhibits proliferation and promotes osteogenesis in hBMSCs by regulating the ERK1/2 signaling pathway. Sci Rep 7: 10342, 2017.

37. Chen FM, Chen R, Wang XJ, Sun HH and Wu ZF: In vitro cellular responses to scaffolds containing two microencapulated growth factors. Biomaterials 30: 5215-5224, 2009.

38. Koo T, Park SW, Jo DH, Kim D, Kim JH, Cho HY, Kim J, Kim JH and Kim JS: CRISPR-LbCpf1 prevents choroidal neovascularization in a mouse model of age-related macular degeneration. Nat Commun 9: 1855, 2018.

39. Du J, Xie P, Lin S, Wu Y, Zeng D, Li Y and Jiang X: Time-Phase sequential utilization of adipose-derived mesenchymal stem cells on mesoporous bioactive glass for restoration of critical size bone defects. ACS Appl Mater Interfaces 10: 28340-28350, 2018

40. Deng L, Hu G, Jin L, Wang $\mathrm{C}$ and Niu H: Involvement of microRNA-23b in TNF- $\alpha$-reduced BMSC osteogenic differentiation via targeting runx2. J Bone Miner Metab 36: 648-660, 2018.

41. Kim KM and Jang WG: Zaluzanin C (ZC) induces osteoblast differentiation through regulating of osteogenic genes expressions in early stage of differentiation. Bioorg Med Chem Lett 27: 4789-4793, 2017

42. Wang Z, Tan J, Lei L, Sun W, Wu Y, Ding P and Chen L: The positive effects of secreting cytokines IL-17 and IFN- $\gamma$ on the early-stage differentiation and negative effects on the calcification of primary osteoblasts in vitro. Int Immunopharmacol 57: 1-10, 2018.
43. Han Y, Jin Y, Lee SH, Khadka DB, Cho WJ and Lee KY: Berberine bioisostere Q8 compound stimulates osteoblast differentiation and function in vitro. Pharmacol Res 119: 463-475, 2017.

44. Qiao M, Shapiro P, Kumar R and Passaniti A: Insulin-like growth factor-1 regulates endogenous RUNX2 activity in endothelial cells through a phosphatidylinositol 3-kinase/ERK-dependent and Akt-independent signaling pathway. J Biol Chem 279: 42709-42718, 2004.

45. Heldin J, O'Callaghan P, Hernández Vera R, Fuchs PF, Gerwins $P$ and Kreuger J: FGD5 sustains vascular endothelial growth factor A (VEGFA) signaling through inhibition of proteasome-mediated VEGF receptor 2 degradation. Cell Signal 40: 125-132, 2017

46. Gurkan A, Tekdal GP, Bostanci N and Belibasakis GN: Cytokine, chemokine, and growth factor levels in peri-implant sulcus during wound healing and osseointegration after piezosurgical versus conventional implant site preparation: Randomized, controlled, split-mouth trial. J Periodontol 90: 616-626, 2019.

47. Lin S, Zhang Q, Shao X, Zhang T, Xue C, Shi S, Zhao D and Lin Y: IGF-1 promotes angiogenesis in endothelial cells/adipose-derived stem cells coculture system with activation of PI3K/Akt signal pathway. Cell Prolif 50: 2017.

48. Wan Y, Zhuo N, Li Y, Zhao W and Jiang D: Autophagy promotes osteogenic differentiation of human bone marrow mesenchymal stem cell derived from osteoporotic vertebrae. Biochem Biophys Res Commun. 488: 46-52, 2017.

49. Pantovic A, Krstic A, Janjetovic K, Kocic J, Harhaji-Trajkovic L, Bugarski D and Trajkovic V: Coordinated time-dependent modulation of AMPK/Akt/mTOR signaling and autophagy controls osteogenic differentiation of human mesenchymal stem cells. Bone 52: 524-531, 2013 